\title{
Modelling the seasonal dynamics of SPM with a simple algorithm for the buffering of fines in a sandy seabed
}

\author{
Thijs VAN KESSEL ${ }^{1}$, Han WINTERWERP ${ }^{1,2}$, Bram VAN PROOIJEN ${ }^{3,2}$, \\ Mathijs VAN LEDDEN ${ }^{4}$ and Wil BORST ${ }^{5}$ \\ 1. WL | Delft Hydraulics, P.O. Box 177, 2600 MH Delft, The Netherlands. \\ Email: thijs.vankessel@wldelft.nl
}

2. Delft University of Technology, P.O. Box 5048, 2600 GA Delft, The Netherlands Email: j.c.winterwerp@tudelft.nl 3. Svasek Hydraulics, Schiehaven 13G, 3024 EC Rotterdam, The Netherlands Email: b.c.vanprooijen@tudelft.nl

4. Royal Haskoning, Postbus 151, 6500 AD Nijmegen, The Netherlands Email: m.vanledden@haskoning.com

5. Port of Rotterdam, P.O. Box 6622, 3002 AP Rotterdam, The Netherlands Email:.wg.borst@portofrotterdam.com

Keywords: suspended sediment, 3D model, seasonal dynamics, North Sea, bed algorithm.

\begin{abstract}
This paper discusses the application of a simple algorithm for the buffering of fines in a sandy seabed. A second layer is introduced in which fines may be stored during calm weather and from which fines may be resuspended during storms. The algorithm is applied first in a one-dimensional vertical (1DV) point model at a location in the North Sea, Noordwijk 10, $10 \mathrm{~km}$ offshore. It is able to reproduce the observed temporal variability of suspended particulate matter satisfactorily. Apart from the second layer, also the applied first order erosion rate is an important element of the algorithm. This allows for an equilibrium sediment mass per unit area for any combination of bed shear stress climate and sediment supply. The classical Partheniades-Krone formulation with zero ${ }^{\text {th }}-$
\end{abstract}


order erosion (i.e. an erosion rate that is independent from the sediment mass per unit area) does not have such equilibrium.

As a next step, the algorithm is incorporated into a 3D model for suspended particulate matter (SPM) transport in the Dutch coastal zone. It is demonstrated that the model is able to reproduce the observed spatial and temporal variability reasonably well. An essential feature of the 3D mud model is that it is sufficiently fast to compute equilibrium bed composition. This implies that the results are completely independent from the applied (uniform) initial conditions.

Finally, the mud model is applied to assess the impact of a large-scale release of fines in the Dutch coastal zone. The computed impact turns out to be very sensitive to the assumed buffer capacity of the seabed. However, information on transient system response (such as the dissipation of a sediment pulse in the system) from which the buffer capacity may be estimated is most often lacking. For the time being, estimates on the residence time of fines in the seabed and its mixing depth are derived from the literature. Additional field and laboratory test on the exchange mechanisms of fines between the water column and a sandy seabed are recommended.

\section{Introduction}

The concentration of suspended particulate matter (SPM) along the Dutch coast varies strongly on a short time scale because of wave and tidal action, but also on a seasonal scale. The wave climate in summer is milder than in winter, and also sediment supply may be different. The observed widespread sudden SPM concentration increase during storms demonstrates that on a short timescale, vertical exchange of sediment between the sea bed and the water column is dominant. A simple new bed algorithm was developed to capture the observed SPM variations in the Dutch coastal zone into a numerical model. In this paper, the formulations of the bed algorithm are discussed first. Secondly, the algorithm is applied in a 1DV point model, describing vertical exchange only. Subsequently, the algorithm is also applied in a 3D mud transport model of the southern North Sea, focusing on the Dutch coastal zone (Figure 1). Two types of results are discussed: 1) modelling of the natural background SPM levels and 2) modelling the impact of an additional release of fines, e.g. originating from sand mining. Throughout the paper, the results 
obtained with the new algorithm are compared with results from a classical algorithm. Differences between the classical and novel approach are discussed. The paper is concluded with a summary of the most essential implications of the use of the new bed algorithm in mud models.

\section{Algorithm for buffering of fines in a sandy seabed}

The bed algorithm models the seabed into two layers, see Figure 2. The first layer is representative for the thin fluff layer on the bed surface that forms during slack tide and that is easily resuspended by tidal currents. The total sediment mass in this layer tends to be small and the residence time of sediment in this layer is short because of the large flux between the fluff layer and the water column. The second layer is representative for the sandy seabed into which fines may entrain and temporarily be stored. Resuspension from this buffer layer is only significant during highly dynamic conditions, such as spring tide or storms. The time-average sediment flux between the buffer layer and the water column is therefore limited, whereas the storage capacity of fines in the seabed may be large - depending on the assumed mixing depth. As a result, the residence time of fines in the sandy seabed may be large. According to an estimate by Laane et al. (1999), this residence time may be in the order of two years.

The formulations for the sediment exchange between the bed layers and the water column are:

- Deposition flux towards layers 1 and 2: $D_{1}=(1-\alpha) w_{s} C ; D_{2}=\alpha w_{s} C$

- Resuspension flux from layers 1 and 2: $E_{1}=m_{1} M_{1}\left(\tau / \tau_{\mathrm{cr}, 1}-1\right) ; E_{2}=p_{2} M_{2}\left(\tau / \tau_{\mathrm{cr}, 2}-1\right)^{1.5}$

where $w_{s}$ is settling velocity, $C$ is near-bed SPM concentration, $\alpha$ is the fraction of the deposition flux contributing to layer $2(\alpha<<1), \tau_{\mathrm{cr}, i}$ the critical shear stress for erosion for layer $i\left(\tau_{\mathrm{cr}, 1}<\tau_{\mathrm{cr}, 2}\right)$, $M_{i}$ the resuspension parameter for layer $i, m_{1}$ the sediment mass per unit area in layer 1 and $p_{2}$ the fines fraction in layer 2. These formulations are also discussed by Van Prooijen et al. (2007).

The exponent 1.5 in (2) stems from Van Rijn's (1993) empirical pick-up function for a sandy seabed. The parameter $m_{1}$ in (2) transforms the zeroth order resuspension rate according to Partheniades in to a first order resuspension rate, i.e. the resuspension rate scales linearly with the sediment mass per unit area in layer 1 . The rationale for this modification is that the expression by 
Partheniades is valid for a $100 \%$ mud bed, whereas along the Dutch coast the fluff layer probably does not completely cover the sandy seabed, but tends to accumulate in the troughs of sand ripples and sand waves. A first order resuspension rate may be more realistic in this case.

Compared to some other recent formulations for water-bed exchange of sediment (e.g. Warner et al., 2008; Gayer et al., 2006), the bed may become depleted of fines applying the present formulations. Warner et al. (2008) and Gayer et al. (2006) describe a bed model in which the 'active layer' of erodible material is replenished from below. Another difference is that in the present formulations the erosion rate of each sediment fraction in fluff layer 1 scales with its absolute availability (in $\mathrm{kg} / \mathrm{m}^{2}$ ) instead of its relative availability, i.e. sediment fraction (-). The erosion rate therefore is first order instead of zeroth order.

The application of the first order erosion rate results in a linearly scalable solution for SPM concentration, i.e. different sediment fractions or sources behave independently and may be added or removed arbitrarily. This allows for the computation of the dispersion of a point source of fines without the necessity to take background SPM concentration into account, for example. The second bed layer is essential to introduce multiple response timescales, which are also observed from nature. Also, a specific storm event may show a different SPM concentration response, depending on the local shear stress history and sediment supply. These factors determine how much sediment is locally available and how this sediment is distributed over the water column, the fluff layer and the sandy seabed.

The first order erosion rate implies that net permanent deposition is impossible with these formulations. After all, mud deposition will result in an increase of $m_{1}$ or $p_{2}$ in (2) until a mass per unit area is reached for which erosion equals deposition. Therefore in environments with a higher mud supply the erosion term could be modified into $E_{1}=\min \left(m_{1} M_{1}, M_{0}\right)\left(\tau / \tau_{\mathrm{cr}, 1}-1\right)$, assuming a transition from first order to zeroth order erosion at $m_{1}=M_{0} / M_{1} \mathrm{~kg} / \mathrm{m}^{2}$. The rationale behind this transition is that for small values of $m_{1}$ the exposed surface area of mud may scale linearly with $m_{1}$, 
as the seabed is not yet completely covered with mud. However, at a certain value of $m_{1}$ the seabed will become completely covered with mud and even more mud will not enhance the erosion rate anymore. Note that linear scalability is lost by introducing this transition. To avoid a completely muddy first layer on top of a sandy second layer, $M_{2}$ should not be chosen larger than about $\alpha M_{0}$.

The advantage of the novel approach is that the model behaviour becomes smoother, as for any combination of sediment supply and local bed shear stress climate an equilibrium bed composition exists. With the Partheniades-Krone formulations no equilibrium exists: given the bed shear stress climate and sediment supply the sediment mass on the bed per unit area becomes always zero or infinity on the long term.

The buffer capacity $B$ is defined as the mass per unit area stored in the sandy seabed (layer 2 ) under equilibrium conditions regarding sediment supply and local bed shear stress climate. It is computed according to: $B=p_{2, \mathrm{eq}}(1-n) \rho_{\mathrm{s}} d_{2}$, where $d_{2}$ is the thickness of layer $2, n$ its porosity and $\rho_{\mathrm{s}}$ the solids density of sand (about $2650 \mathrm{~kg} / \mathrm{m}^{3}$ ). The equilibrium mud fraction $p_{2, \mathrm{eq}}$ follows from a balance between settling and resuspension:

$$
D_{2}=E_{2} \Leftrightarrow p_{2, \mathrm{eq}}=\frac{\alpha w_{s} \bar{C}}{M_{2} \int_{t} \max \left(0,\left(\frac{\tau}{\tau_{\mathrm{cr}, 2}}-1\right)\right)^{1.5}}
$$

The buffer capacity can be chosen by the user either by varying $d_{2}$ (which does not have consequences for the resuspension flux unless $d_{2}$ is sufficiently small to result in a depletion of fines) or by varying $w_{s}, M_{2}$ or $\tau_{\mathrm{cr}, 2}$ (which does have consequences for the settling and resuspension fluxes).

Coefficient $\alpha$ steers the infiltration rate of fines into the sandy seabed. One possible infiltration mechanism is the wave- and tide-induced infiltration of seawater into the seabed. Fines present in the seawater are filtered out and remain in the seabed. Other mechanisms may also be important, such as bioturbation. Erosion of mud from the sandy seabed is only possible for conditions in 
which the sandy seabed is eroded, which explains the exponent 1.5 in (2). However, it is remarked that sand transport is not modelled with the present approach. Only the resuspension of fines from the seabed is taken into account. Typically, the value for $\tau_{\mathrm{cr}, 2}$ is set at a value that allows for resuspension of fines only during spring tide and storms. A further explanation on the calibration of the $2 \mathrm{~L}$ model is given in the next section.

\section{1DV point model}

The new bed algorithm was implemented into a 1DV model, which has been used to study the model behaviour and calibrate the parameter settings with a data set acquired at Noordwijk $10 \mathrm{~km}$ offshore. This dataset consist of the hourly averaged SPM surface concentration data in the period 1/1 - 18/9 2001 (Hartog en Van de Kreeke, 2003). By definition the 1DV model has zero horizontal gradients. The user-defined initial sediment mass per unit area therefore remains constant, as the inward flux equals the outward flux. However, the distribution of sediment among the water column and the first and second bed layer varies in time, depending on the forcing by tide and waves. Alternatively, a 2DV model may be applied with the observed year and depth-averaged SPM level at Noordwijk $10 \mathrm{~km}$ as boundary concentration $(7.5 \mathrm{mg} / \mathrm{l})$. For a sufficiently long channel, results at mid-length become identical to those obtained with the 1DV closed model.

Figure 3 shows both the observed SPM concentration at Noordwijk $10 \mathrm{~km}$ and the concentration computed with the 1DV model. The bed shear stress $\tau_{\text {bed }}$ forcing the water-bed exchange of sediment is shown on the right hand side $y$-axis. The $1 \mathrm{DV}$ model consists of 10 layers in the water vertical. The velocity profile is assumed to be logarithmic. Applied bed roughness is $0.1 \mathrm{~m}$. Local water depth is $18 \mathrm{~m}$. Vertical diffusion is set at a constant value of $\varepsilon_{s}=10^{-2} \mathrm{~m}^{2} / \mathrm{s}$, estimated from $\varepsilon_{s}$ $=(1 / 4) \kappa h \sqrt{ }\left(\tau_{\text {bed }} / \rho\right)$, where $h$ is water depth and $\rho$ is water density. This simple approach is chosen as the 1DV model is not meant to reproduce observed vertical salinity gradients at Noordwijk $10 \mathrm{~km}$, which have a strong 3D character. In the 3D model, the effect of salinity gradients on the vertical diffusion is taken into account with a $k$ - $\varepsilon$ turbulence model. Computed vertical diffusion is variable, but in the order of $10^{-2} \mathrm{~m}^{2} / \mathrm{s}$ applied in the 1DV model. Based on Figure 3, it is concluded that the 
new bed algorithm is capable to reproducing the observed temporal variability satisfactorily. Both short-term fluctuations and long-term trends are reproduced.

Table 1 shows the parameter settings as established with the 1DV simulations. Two fractions are included to reproduce the observed vertical sediment concentration gradients during storm conditions and the observed baseline concentration during calm weather, when little vertical gradients are observed.

From Table 1 it is concluded that during the calibration 7 parameters for each of the 2 fractions should be optimised. However, this turns out to be less tedious than it may appear at first sight. The settling velocity of the finest fraction is optimised from the observed concentration decay after storms. The settling velocity of the coarser fraction is optimised from the observed vertical concentration gradient during storms. The critical shear stress for erosion of layer 1 is derived from the observed onset of erosion of the fluff layer after slack water. The first-order resuspension parameter $M_{1}$ for both fractions is chosen such that the tidal fluctuation of the observed SPM concentration is reproduced well. Note that the calibration is quite insensitive to the chosen value of $M_{1}$, but it should be set within a range that results in a sufficient equilibrium mass per unit area to be able to reproduce the observed tidal SPM fluctuations (typically $\Delta C / h \mathrm{~kg} / \mathrm{m}^{2}$, where $\Delta C$ is the observed tidal concentration fluctuation and $\mathrm{h}$ is water depth). However, it should not be chosen so small that the assumption of a first order erosion rate is violated and the fluff layer starts acting as a significant sediment buffer compared with the buffer capacity of layer 2.

Regarding the parameter settings for layer $2, \tau_{\mathrm{cr}, 2}$ follows from the properties of the sandy seabed such as $D_{50}$. The resuspension parameter $M_{2}$ is chosen to match the observed peaks in SPM levels during storms. Parameter $\alpha$ is chosen to match the computed equilibrium mud fraction in the seabed with the observed mud fraction for a typical year-averaged SPM level $\bar{C}$ according to (3). Finally, layer thickness $d_{2}$ is chosen to match to measured (or assumed) residence time of fines in the seabed, thus establishing the buffer capacity $B$. Only a single set of parameter values exists for 
layer 2 that meets the demand on storm-induced concentration peak height, equilibrium mud fraction and mean residence time in the seabed. The formulations (1) and (2) therefore do not contain redundant parameters. However, the set of parameter values obtained after calibration is robust in the sense that small changes in parameter values result in small changes of computed SPM levels only.

Figure 4 shows the modelled SPM variation at Noordwijk 10 for different bed formulations. The novel $2 \mathrm{~L}$ approach is shown in blue, the $1 \mathrm{~L}$ approach with first order erosion in green and the classical 1L approach with zeroth order erosion in red. Scatter plots of modelled versus observed SPM concentration are shown in Figure 5 for the $2 \mathrm{~L}$ and classical $1 \mathrm{~L}$ approaches. Although the results of the three approaches seem quite similar at first sight, three fundamental observations are made:

1. The $2 \mathrm{~L}$ approach results in the highest storm concentration peaks, which is agreement with observations on typical SPM storm levels. Storm peaks and seasonal dynamics in general are less pronounced for a $1 \mathrm{~L}$ approach. The improved match of modelled and observed concentration peaks results in a higher correlation between model results and observations, see Figure 5.

2. The calibration result shown in Figure 3 can de obtained with arbitrary second layer depth $d_{2}$. This implies that additional information is required on the residence time of fines in the seabed and the total active mass of fines in the vertical per unit area (both suspended and in the seabed). This is a dominant factor for transient conditions such as the system's response to a sudden 'shock' like the sudden release of a substantial amount of sediment into the system.

3. The results for the classical zeroth order approach are very sensitive to the applied parameter settings. The red line in Figure 4 is obtained assuming a balance between sedimentation and erosion over the year. However, when the settings are changed slightly, the computed SPM levels change drastically. This is illustrated in Figure 6. Although the 
red line is similar to the computed SPM levels for a first order approach (see Figure 4), the computed SPM levels change drastically for small changes in the zeroth order erosion parameter $M_{0}$. An increase in $M_{0}$ results in depleted bed conditions and a flat SPM signal (as sediment is nearly permanently in suspension), whereas a decrease in $M_{0}$ results in continuous sediment accumulation on the bed and a concurrent strong decrease of the SPM level. This explains why it is difficult to calibrate a fine sediment model with classical erosion formulations. For a first order erosion formulation, computed SPM levels are much less sensitive to parameter settings.

\section{3D model for Southern North Sea}

The next step is to apply the 2 layer approach to a 3D model of the southern North Sea, the ZUNO model (Figure 7). The number of grid cells is approximately 4500. The resolution of the coarse grid variant applied in the present study varies from quite coarse far offshore (10 km resolution) to finer close to the Dutch coast (2 $\mathrm{km}$ resolution). The coarse grid variant is applied to make long simulation periods possible exceeding the residence time of fines in the system. The ZUNO-coarse model has 10 vertical $\sigma$-layers with higher vertical resolution in the areas where maximum velocity shear is expected, i.e. near-bottom for the tidal flow and near-surface for wind forcing. From bottom to surface, 10 horizontal layers have been applied with a relative thickness of 4.0, 5.9, 8.7, $12.7,18.7,18.7,12.7,8.7,5.9$, and $4.0 \%$ of the total water depth. The relative thickness of the surface layer and of the bottom layer is $4 \%$, i.e. $0.8 \mathrm{~m}$ at a depth of $20 \mathrm{~m}$.

The hydrodynamic simulations are forced with a representative tide based on 50 astronomic components, and the actual rivers discharges, wind and atmospheric pressure. The hydrodynamic simulations cover a period of 8 years, from 1996 until 2003. The hydrodynamic time step is set at 5 minutes, which is sufficiently small, considering the spatial resolution, to obtain accurate model results. For details of the hydrodynamic simulations the reader is referred to De Goede and Van Maren (2005). 
The SPM model grid is identical to the ZUNO-coarse hydrodynamic grid, without any aggregation, covering the complete southern part of the North Sea. Also the SPM transport simulations cover a period of 8 years. For most simulations, the hydrodynamic year 2000 has been repeated 8 times, but for some simulations the complete hydrodynamic database from 1996 to 2003 has been utilised. The rationale behind the repeated use of a single year of hydrodynamics is that this allows for a better judgement on the model spin-up time required to attain a dynamic equilibrium. The large inter-annual variability complicates such a judgement when using eight successive years.

The mud model is forced as follows: southern boundary concentration $6 \mathrm{mg} / \mathrm{l}$; northern boundary concentration $2 \mathrm{mg} / \mathrm{l}$; river loads $3.7 \mathrm{MT} / \mathrm{y}$ (to which the New Waterway near Rotterdam contributes most with $675 \mathrm{kT} / \mathrm{y}$ ); coastal erosion 10.8 MT/y (Holderness, Norfolk, Suffolk, Channel) and bed erosion (Flemish Banks) 2.0 MT/y. The load through Dover Strait depends on the hydrodynamic forcing and ranges between 25 and 46 MT/y. This flux therefore dominates the system. These loads are similar as those reported in Gerritsen et al. (2000) and Gerritsen et al. (2001). The applied parameter settings are shown in Table 1.

Wave-induced resuspension is modelled with a wave forcing from the wave propagation model SWAN, or with an assimilated wave field derived from wave observations interpolated with SWAN wave fields.

As initial conditions, a uniform concentration of $6 \mathrm{mg} / \mathrm{l}$ is prescribed in the water column and a uniform mud fraction of 0.02 in the bed. The simulation period is chosen sufficiently long to reach a dynamic equilibrium, i.e. the bed composition becomes independent from the initial conditions. A period of $2 \times 8$ years is required to reach such a dynamic equilibrium. This dynamic equilibrium is shown in Figure 8, which partly resembles the pattern prescribed by Gayer et al. (2006) as initial condition in their mud model for the southern North Sea. Note that a clear correlation is observed between bathymetry and mud fraction. The ability to simulate a sufficiently long period is an 
essential feature of the present mud model, as the computed concentration patters of mud models with a simulation period that is short compared to the typical spin-up time of the bed are strongly influenced by the user-defined initial bed composition.

\subsection{Modelling of the background SPM value}

Figure 9 shows the observed and computed SPM concentration at Noordwijk $10 \mathrm{~km}$. The computed concentration is obtained with the ZUNO-model in contrast with Figure 3, where the computed SPM concentration is obtained with the 1DV model. Although the local model performance of the ZUNO is less good than that of the point model, it should be realised that the ZUNO model's job is much more challenging: only time-average boundary concentrations hundreds of km away from location Noordwijk 10 have been prescribed by the user. The internal redistribution of mud is fully computed by the model. The arrow in Fig. 9 indicates elevated levels of observed SPM in a period without elevated SPM levels according to the ZUNO-model. This is probably caused by instrument failure, as there are no indications from hydrodynamic forcing which explain high values. The regular MWTL observations (indicated in black in Fig. 9) show much lower values in July 2001.

Figure 10 shows the observed and computed concentrations along 4 transects perpendicular to the Dutch coast: Walcheren, Goeree, Noordwijk and Callantsoog (see Fig. 7). The model reproduces typical cross-shore SPM gradients quite well. These gradients are formed because of the Rhine plume, resulting in a near-bed residual current directed towards the coast, thereby trapping sediment.

The $2 \mathrm{~L}$ approach is demonstrated to be essential to capture the seasonal dynamics and storm response of SPM levels. Figure 11 shows the computed 2-month summer and winter averaged SPM concentrations with and without sediment buffering. This figure shows that the modelled seasonal dynamics of SPM are much less pronounced for the $1 \mathrm{~L}$ approach compared to the $2 \mathrm{~L}$ approach. The $2 \mathrm{~L}$ approach is in better agreement with observations along the Dutch coast, see Figure 1. The observations demonstrate that a clear seasonal trend exists in SPM levels, with lower values in summer and higher values in winter. 
The above comparisons between computed and observed SPM levels demonstrate that the $2 \mathrm{~L}$ mud model is capable to reproducing the observed typical spatial and temporal variability in SPM. Work is ongoing to define more objective validation criteria. Also, the model results will be compared with satellite observations on SPM, yielding information on stochastic and systematic errors (Blaas et al., 2007; El Serafy et al., 2009). This will help to improve the model further. For more detailed information is referred to a technical report describing the set-up and calibration of the ZUNO model (Van Kessel and Brière, 2006). Also the mechanisms for infiltration of fines into the seabed and erosion of fines from the seabed should be studied in more detail, both in the field and in the laboratory, as the discussed 2L model still has a 'black box' character.

\subsection{Modelling the large-scale dispersion following a release of fines}

As a next step, the mud model is used to assess the impact of the release of a large volume of fines on the SPM levels in the Dutch coastal zone. Such release may occur because of large-scale sand mining, for example. Only far-field effects are considered here. The main uncertainty in the impact assessment is the buffer capacity of the seabed. If a low buffer capacity is assumed, the relative increase of the amount of fines per unit area in the seabed may be substantial, resulting in substantial relative effects. Substantial effects in the seabed also result in substantial effects in the water column. However, if a high buffer capacity is assumed, the relative increase of fines is smaller and therefore initially also its relative impact. Nonetheless, in the latter case the effect on SPM levels remains discernable for a longer time than in the first case, as the residence time of fines in the system becomes larger for larger buffer capacities.

Figure 12 shows the computed increase in SPM levels after 6 years of sediment release at a rate of 2 MT/y, which is substantial compared to the estimated longshore SPM flux of approximately 10 MT/y. The upper panel shows the increase with a $1 \mathrm{~L}$ approach (i.e. without buffer capacity), the lower panel shows the increase for a $2 \mathrm{~L}$ approach with the same settings for the first layer. It is 
obvious that the relative increase is smaller in case of a larger buffer capacity. For the 1L approach, a concentration increase of 5 to $10 \mathrm{mg} / \mathrm{l}$ is computed in a substantial part of the Dutch coastal zone. However, for the $2 \mathrm{~L}$ approach the computed concentration increase in a zone of similar size is only 2 to $5 \mathrm{mg} / \mathrm{l}$. Estimates on buffer capacity can not be easily derived from SPM measurements, as different capacities give similar calibration results on the short term. The best estimate on buffer capacity is a residence time of 2 years in the seabed (Laane et al., 1999) and a layer thickness of 30 $\mathrm{cm}$ (equivalent with $5 \mathrm{~kg} / \mathrm{m}^{2}$ fines for a mud fraction of 0.003 ). Further work is required to reduce the uncertainty regarding buffer capacity. Water quality parameters such as $\mathrm{PCB}, \mathrm{Pb}$ or $\mathrm{Cs}$ levels may yield more data on the residence time distribution of fines in the seabed.

\section{Conclusions}

A new bed algorithm is demonstrated to perform well on the Noordwijk 10 dataset, which has been acquired at the North Sea $10 \mathrm{~km}$ offshore during the year 2001. A 2L approach is required to satisfactorily reproduce observed storm response and seasonal dynamics. Application of a first order erosion rate (that may change into a zero order erosion rate for a high mass per unit area) results in a more stable model response, as for any combination of bed shear stress climate and sediment supply an equilibrium bed composition exists. With the classical Partheniades-Krone zero-order erosion rate such an equilibrium does not exist: the equilibrium sediment mass is either zero for regions with a low sediment supply and/or a high average bed shear stress, or infinity for regions with a high sediment supply and/or a low average bed shear stress. This is a fundamental limitation of the classical expressions for erosion.

Next, the algorithm has been incorporated into a 3D model for SPM transport in the Dutch coastal zone. Computed results have been compared with observations of near-surface and near-bed SPM concentration (including seasonal trends) and seabed composition. Work on comparison with remote sensing images is in progress (Blaas et al., 2007). It is demonstrated that the model is able to reproduce the observed spatial and temporal variability reasonably well. More work is required on a quantitative, objective validation procedure. An essential feature of the $3 \mathrm{D}$ mud model is that it is sufficiently fast to compute equilibrium bed compositions. This implies that the results are 
independent from the initial conditions. All computed spatial patterns are therefore internally generated by the mud model, and not steered by the user (e.g. via the bed composition).

Finally, the mud model is applied to assess the impact of a large-scale release of fines in the Dutch coastal zone (2 MT/y during 6 years). The computed impact turns out to be very sensitive to the applied buffer capacity of the seabed. With a $1 \mathrm{~L}$ model, the computed SPM concentration increase is more substantial than with a $2 \mathrm{~L}$ model, as the equilibrium sediment mass per unit area is higher in the latter case. As a consequence, the same amount of additional sediment therefore has a smaller impact, relatively. A fundamental problem is that under equilibrium conditions, the same calibration result can be obtained with different values for the buffer capacity. Information on transient response (such as the dissipation of a sediment pulse in the system) from which the buffer capacity may be estimated is unfortunately often lacking. For the time being, estimates on the residence time of fines in the seabed and its mixing depth are derived from tracer studies reported in the literature (Laane et al., 1999). Based on these assumptions, it is concluded that the computed SPM concentration increase due to the release of fines remains limited to a value of less than 5 $\mathrm{mg} / \mathrm{l}$ apart from a small nearshore zone close to the release location.

\section{Acknowledgements}

Part of the work presented in this paper was carried out under the project 'Sustainable development of North sea and Coast' (DC-05.20) of the Delft Cluster research project dealing with sustainable use and development of low-lying deltaic areas in general and the translation of specialist knowledge to end users in particular.

\section{References}

Blaas, M., G.Y.H. El Serafy, T. van Kessel, G.J. de Boer, M.A. Eleveld, H.J. van der Woerd. (2007). Data Model Integration of SPM Transport in the Dutch Coastal Zone. Submitted to EUMETSAT conference.

El Serafy, Eleveld, Blaas, Van Kessel, Van der Woerd Assimilating remotely sensed suspended particulate matter in a 3D transport model of the Dutch Coastal Zone. Conditionally accepted for Ocean Modelling. 
Hartog, J, and J. van de Kreeke (2003). Analysis of Optical Back Scatter data observed by the Smart Buoy at the stations Noordwijk 10, Noordwijk 5 and Noordwijk 2. Internal report RIKZ, Ministry of Public Works, the Netherlands.

Gayer, G., S. Dick, A. Pleskachevsky, W. Rosenthal (2006). Numerical modeling of suspended matter transport in the North Sea. Ocean Dynamics 56, pp. 62-77.

Gerritsen, H., R.J. Vos, T. van der Kaaij, A. Lane, J.G. Boon (2000). Suspended sediment modelling in a shelf sea (North Sea). Coastal Engineering 41, pp. 317-352.

Gerritsen, H., J.G. Boon, T. van der Kaaij, R.J. Vos (2001). Integrated Modelling of Suspended Matter in the North Sea. Estuarine, Coastal and Shelf Science 53, pp. 581-594.

Goede E. de, S. van Maren (2005). Impact of Maasvlakte 2 on the Wadden Sea and North Sea coastal zone, Track 1: Deltauled modelling research, Part I: Hydrodynamics. WL | Delft Hydraulics report Z3945.20.

Laane, R., H. Sonneveldt, A. van der Weyden, J. Loch, G. Groeneveld (1999). Trends in the spatial and temporal distribution of metals $(\mathrm{Cd}, \mathrm{Cu}, \mathrm{Zn}$ and $\mathrm{Pb})$ and organic compounds (PCBs and PAHs) in Dutch coastal zone sediments from 1981 to 1996: a model case study for Cd and PCBs. J. Sea Res. 41, pp. 1-17.

Prooijen, B. van, T. van Kessel, M. van Ledden (2007). Modelling of fine sediment in a sandy environment - the coastal zone of the Netherlands. Proc. IAHR 2007 Conference.

Rijn, L.C. (1993). Principles of sediment transport in rivers, estuaries, seas and oceans. Aqua Publications, The Netherlands.

Suijlen, J.M. and Duin R.N.M. (2001). Variability of near-surface total suspended matter concentrations in the Dutch coastal zone of the North Sea. Climatological study on the suspended matter concentration in the North Sea. Report RIKZ/OS/2001.150X.

Van Kessel, T. and Brière, C. (2006). Modelling of seasonal SPM variations in the Dutch coastal zone. WL | Delft Hydraulics Report Z4150.

Warner J.C., C.R. Sherwood, R.P. Signell, C.K. Harris, H.G. Arango (2008). Development of a three-dimensional, regional, coupled wave, current, and sediment-transport model. Computers \& Geosciences 34, pp. 1284-1306. 


\section{Figure captions}

Figure 1: Map of Dutch coastal zone, including observed SPM concentration after stormy weather (left) and summer mean level (right). From silt atlas Suijlen and Duin (2001).

Figure 2: Schematic representation of $2 \mathrm{~L}$ model. Layer $1=$ thin fluff layer; Layer $2=$ sandy seabed infiltrated with fines. $d_{i}=$ thickness of layer $i, D_{i}=$ deposition flux towards layer $i, E_{i}=$ erosion flux from layer $i(i=1,2), C=\mathrm{SPM}$ concentration.

Figure 3: Calibration result with 1DV model for high-frequency data Noordwijk 10, year $=2001$ (labelled 'CEFAS'). Note that data between $6 / 8$ and $21 / 8$ are probably erroneous. The blue line represents the computed SPM concentration (labelled '1DV'). Regular low-frequency observations (labelled 'MWTL') at Noordwijk 10 are also indicated with black stripes. All SPM field and model data are near-surface.

Figure 4: Effect of erosion formulation on computed SPM levels: blue: 2L formulation (also shown in Fig. 2); green: $1 \mathrm{~L}$ formulation, $1^{\text {st }}$ order erosion; red: $1 \mathrm{~L}$ formulation, $0^{\text {th }}$ order erosion $\left(M_{0}=\right.$ $\left.0.5 \times 10^{-6} \mathrm{~g} / \mathrm{m}^{2} / \mathrm{s}\right)$

Figure 5: Modelled versus observed SPM concentration $(\mathrm{mg} / \mathrm{l})$ for $2 \mathrm{~L}$ formulation (left, $R^{2}=0.62$ ) and $1 \mathrm{~L}$ formulation, $0^{\text {th }}$ order erosion (right, $\mathrm{R}^{2}=0.49$ ). $n=5304$. RMS error is $3 \mathrm{mg} / \mathrm{l}$ for both cases. Dotted lines represent factor 2 under- and overestimation. Solid line represents perfect agreement.

Figure 6: Computed SPM concentration with $1 \mathrm{~L}$ and zeroth order erosion rate $M_{0}$. Red line: optimised for equilibrium between sedimentation and erosion $\left(M_{0}=0.5 \times 10^{-6} \mathrm{~kg} / \mathrm{m}^{2} / \mathrm{s}\right.$, also shown in Fig. 3). Purple line: $4 \times$ higher erosion rate (sediment nearly continuously in suspension, depleted 
bed); Blue line $4 \times$ lower erosion rate (sedimentation flux exceeds erosion flux, resulting in net deposition).

Figure 7: ZUNO-coarse grid, the complete southern part of the North Sea.

Figure 8: Equilibrium mud fraction (-) in seabed computed with ZUNO-model. A clear correlation exists between bathymetry and mud fraction with shallower regions showing a lower mud fraction.

Figure 9: Observed and computed (with 3D ZUNO model) SPM concentration (in mg/l) at Noordwijk 10. Compare with 1DV results in Fig. 2. Arrow indicates (probably) erroneous data.

Figure 10: Observed summer and winter mean surface concentration $(\oplus)$. Lines: computed concentration.

Figure 11. Computed 2-month averaged SPM concentration (in $\mathrm{mg} / \mathrm{l})$ is summer $(\mathrm{A}, \mathrm{C})$ and winter (B, D) 2000. A,B: without sediment buffering in sea bed; C,D: with sediment buffering in seabed.

Figure 12: Computed absolute increase in year-averaged SPM surface levels. Upper panel: without buffer capacity; Lower panel : with buffer capacity $5 \mathrm{~kg} / \mathrm{m}^{2}$.

Table 1. Initial sediment parameter settings for the 3D model as determined with the 1DV model. The horizontal dispersion in the $3 \mathrm{D}$ model is set at $\mathrm{D}_{\text {hor }}=1 \mathrm{~m}^{2} / \mathrm{s}$. 

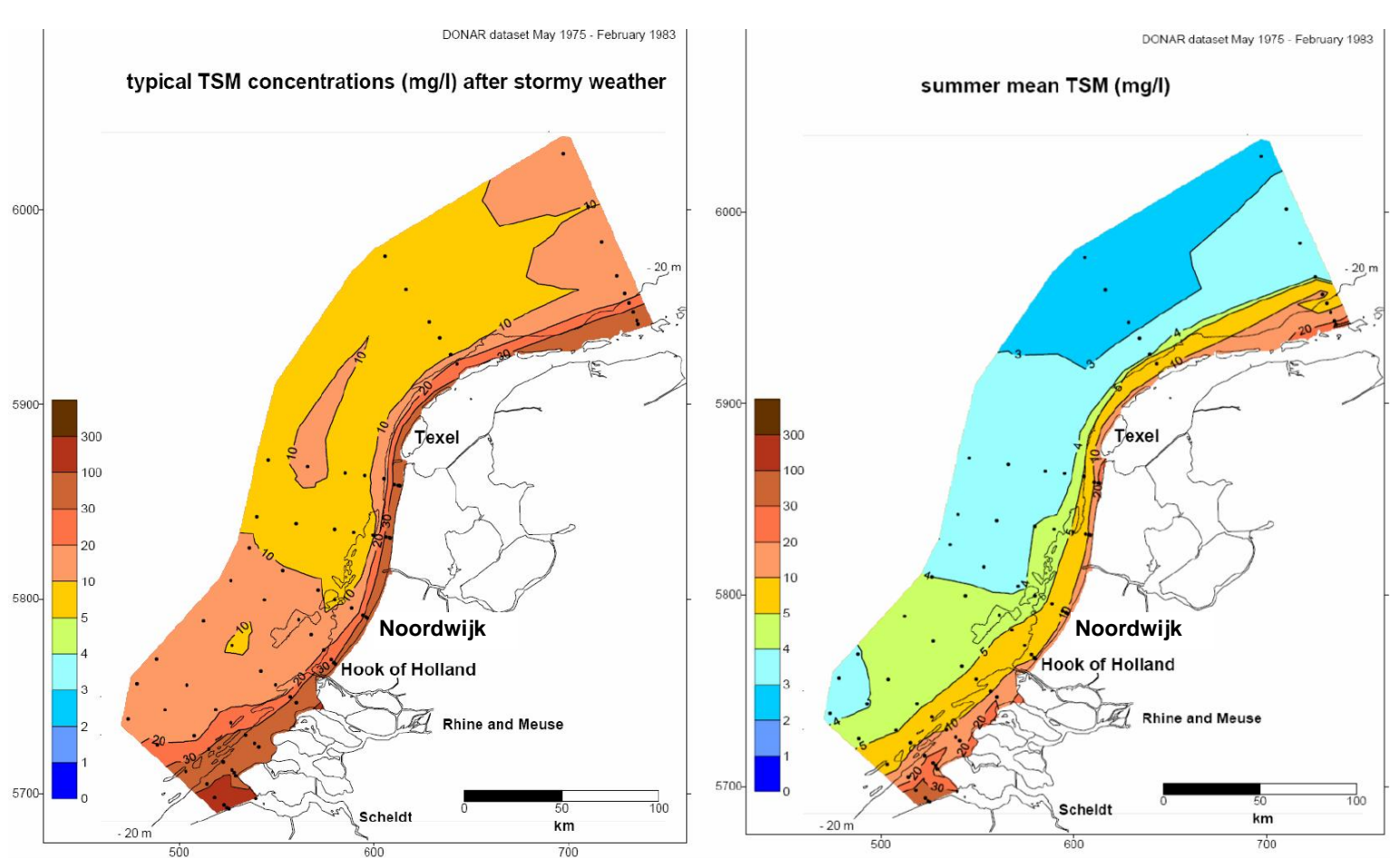

Figure 1 


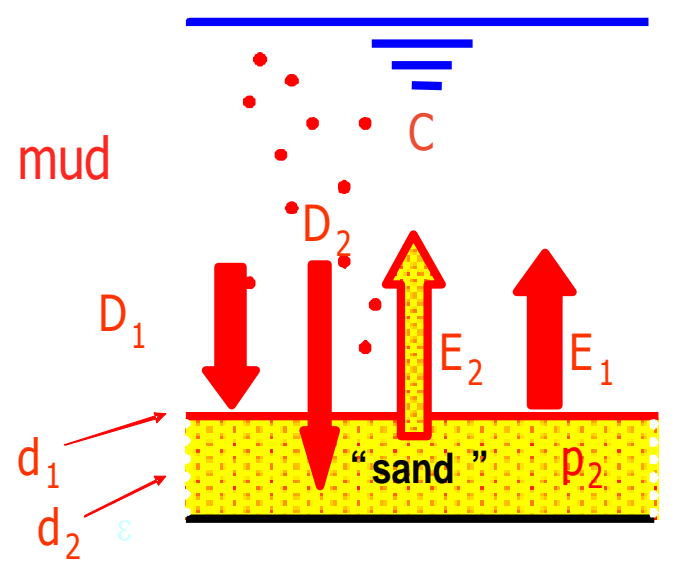

Figure 2 


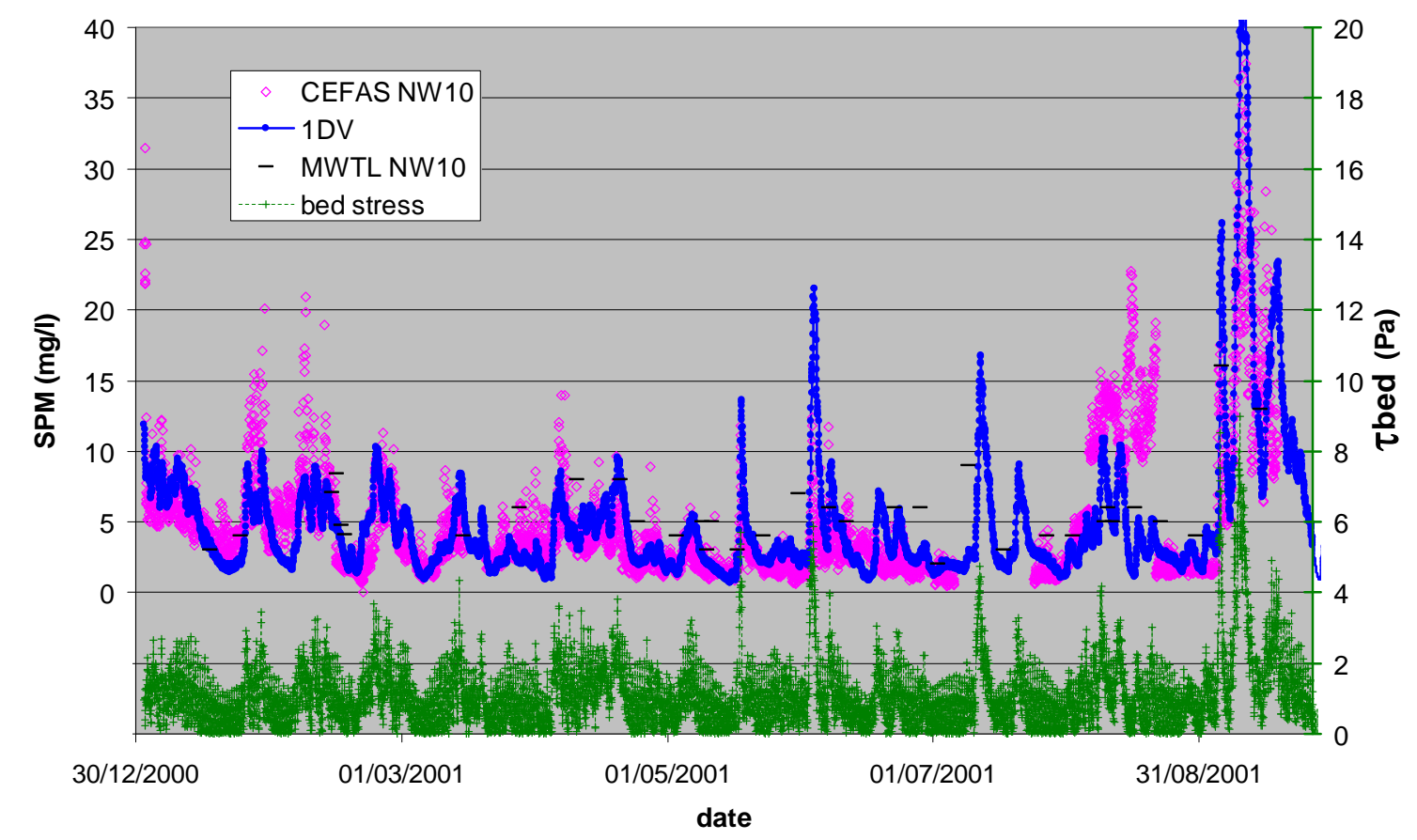

Figure 3 


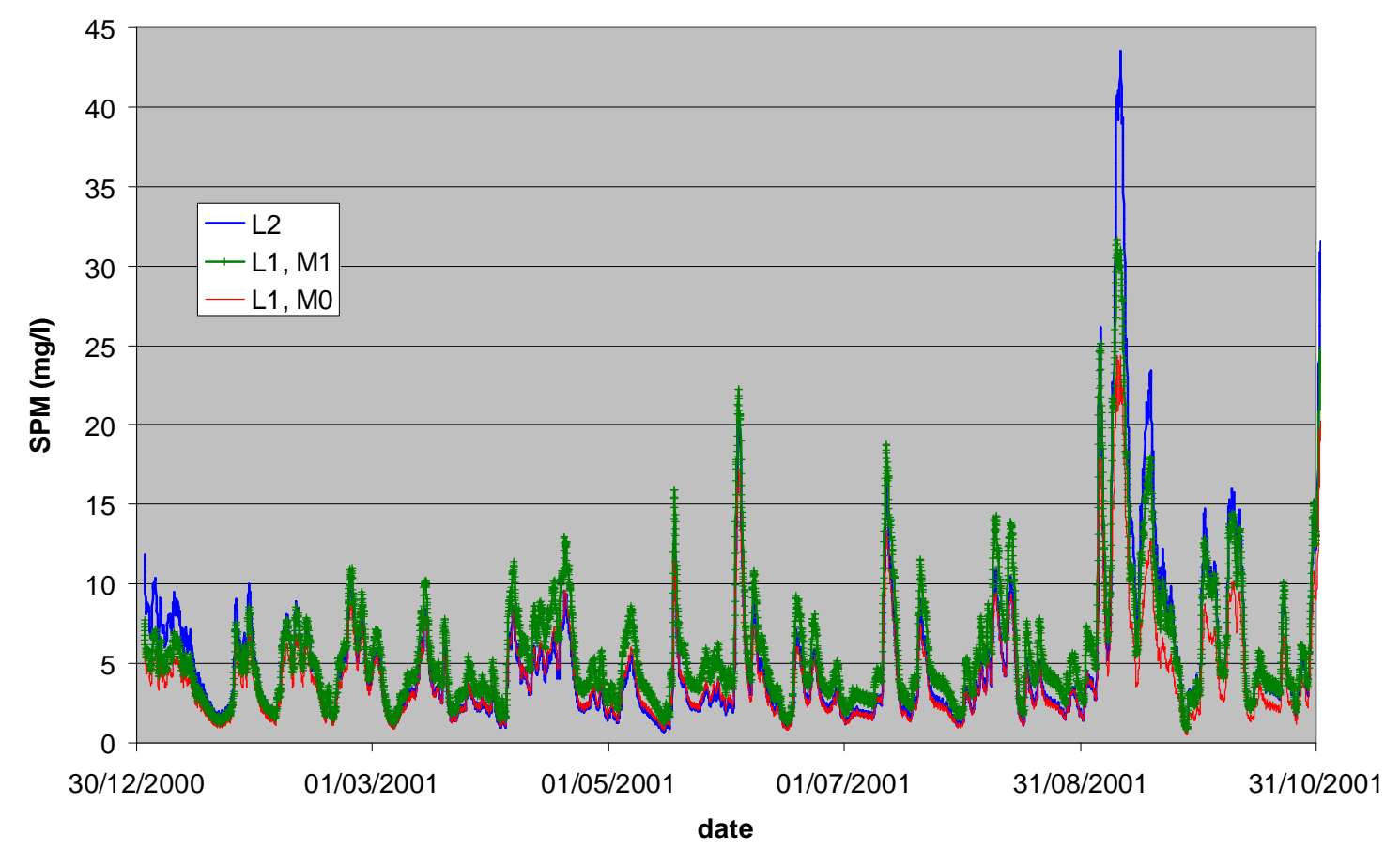

Figure 4 
L2

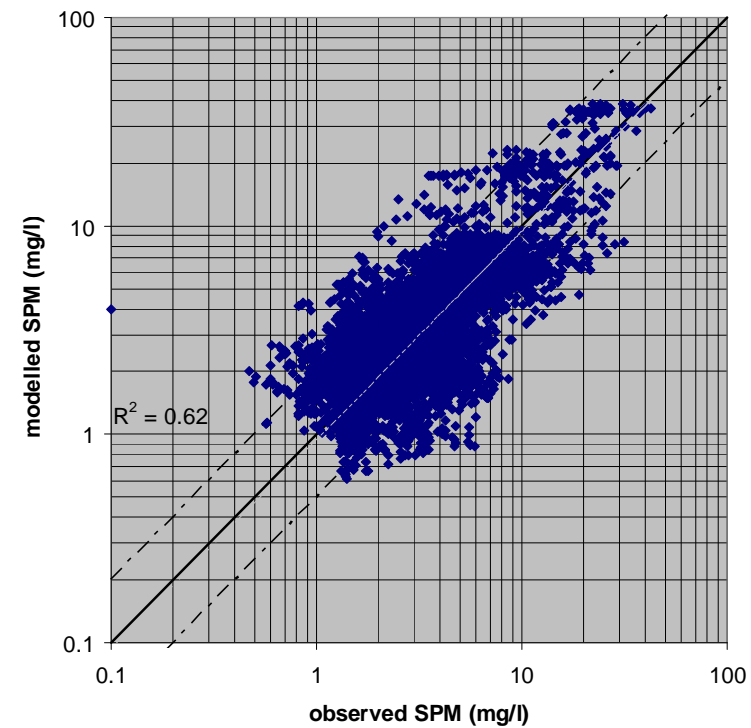

L1, M0

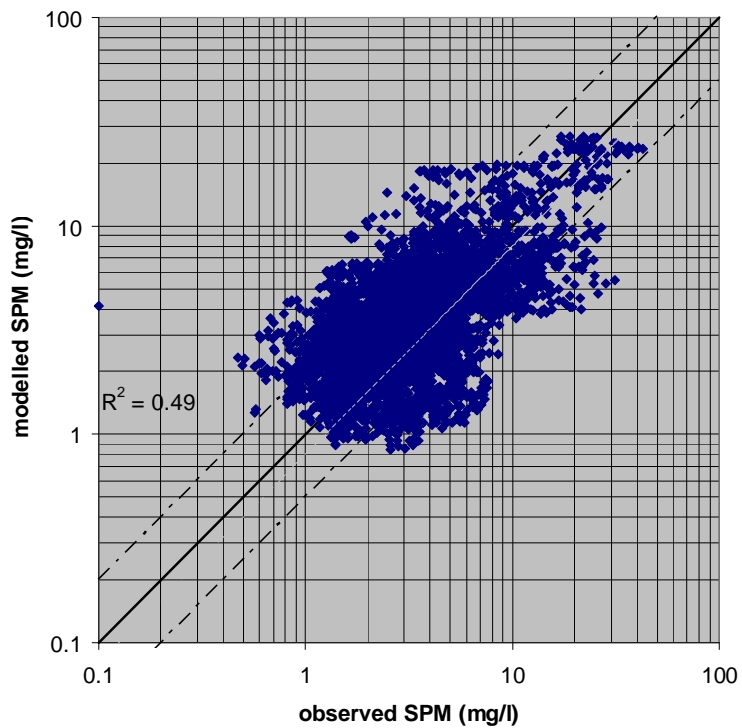

Figure 5 


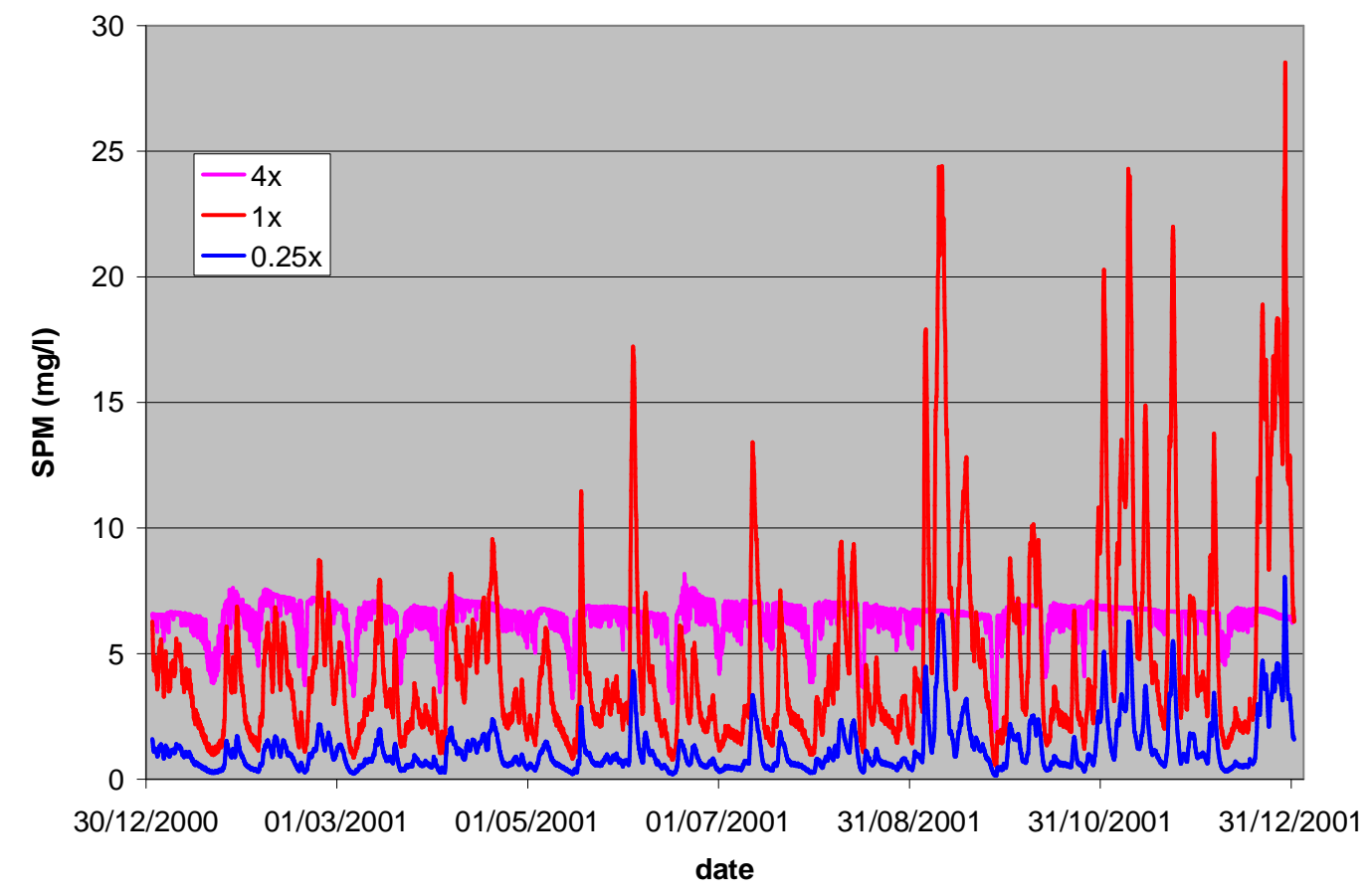

Figure 6 

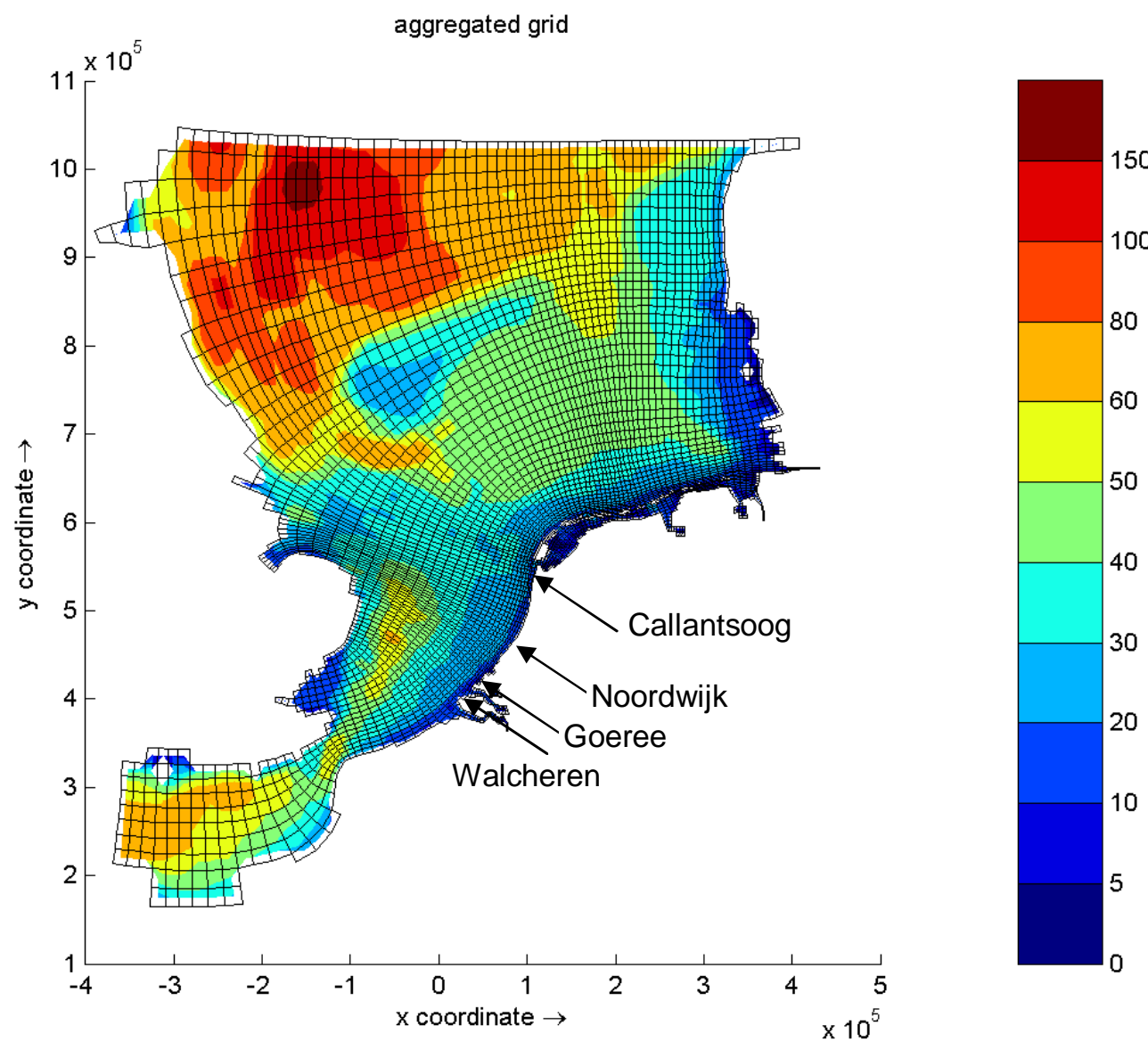

Figure 7 


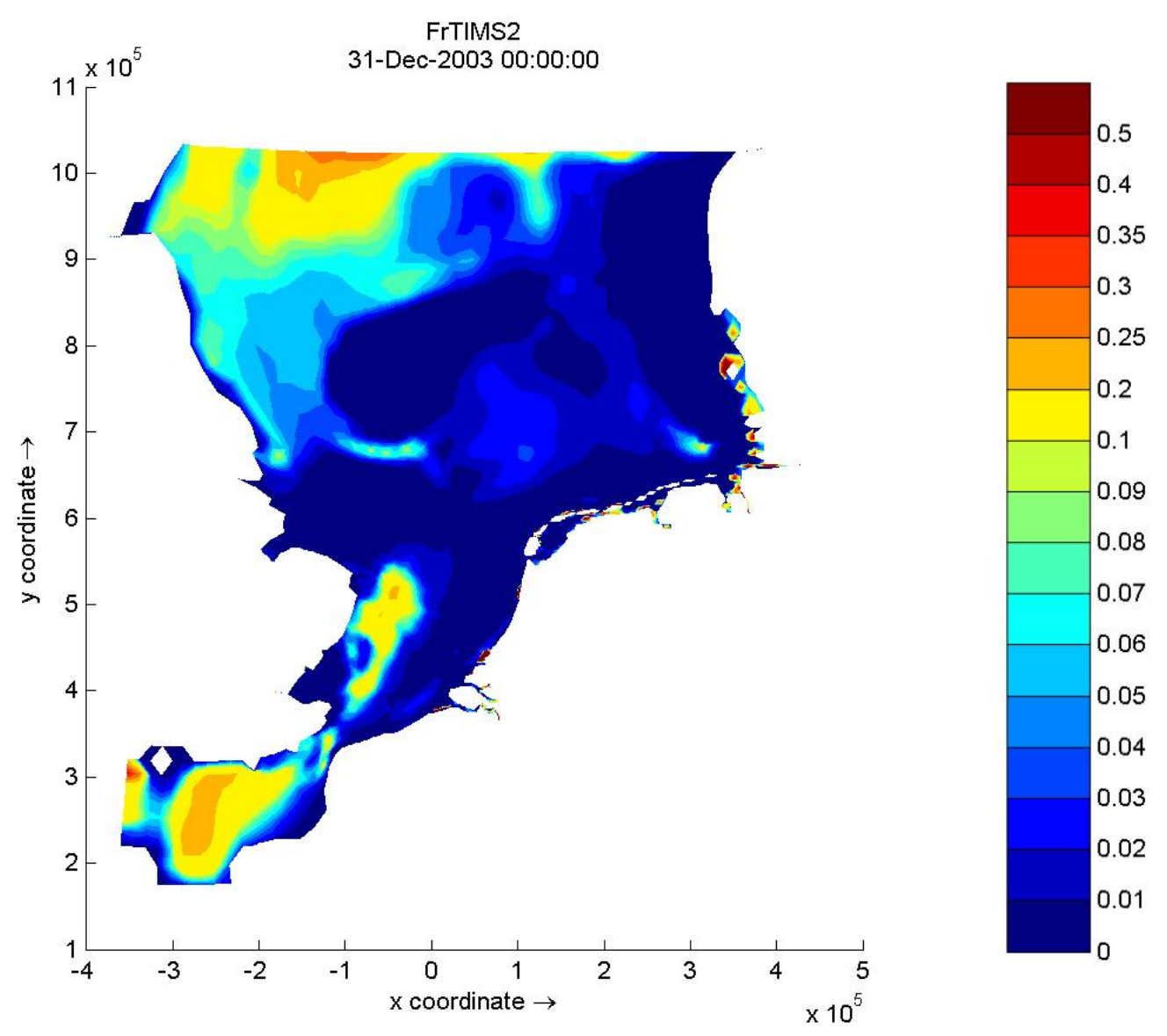

Figure 8 


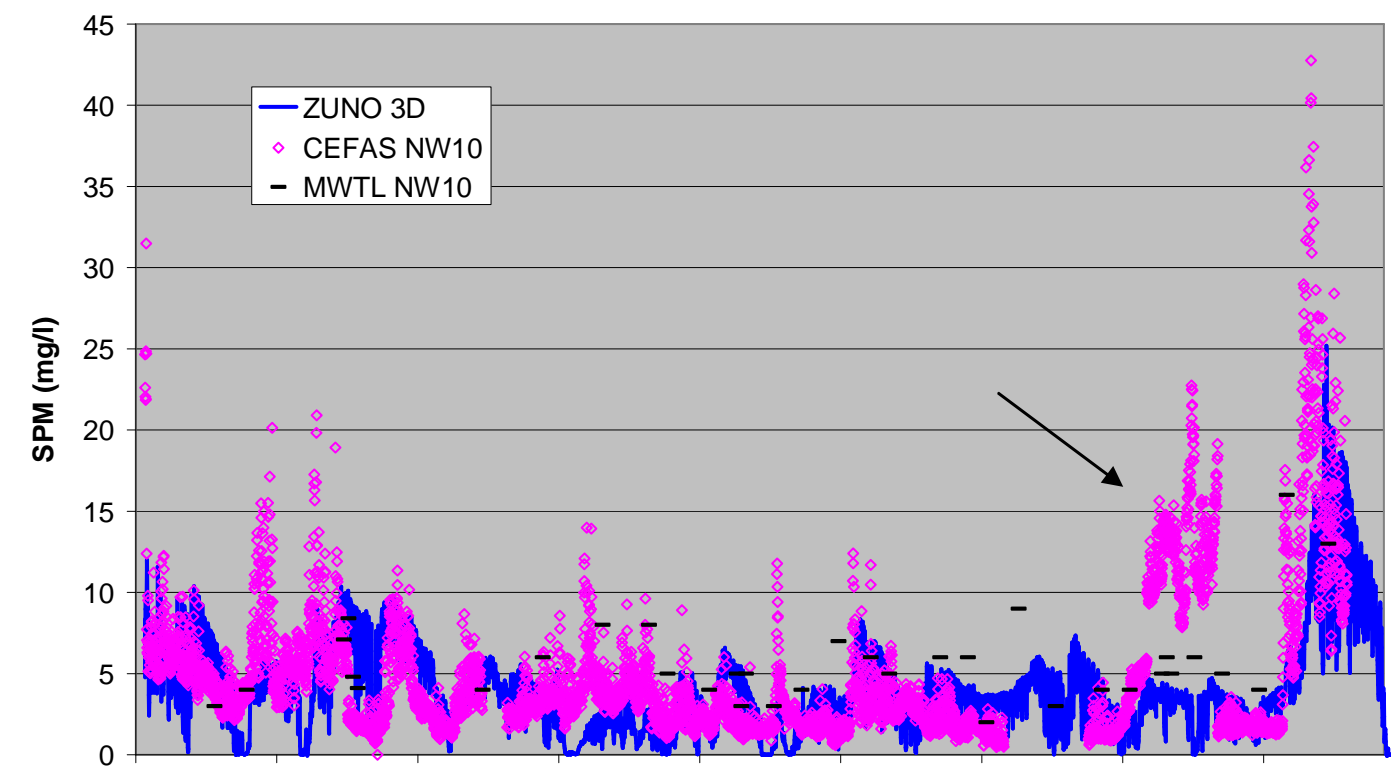

30/12/2000 29/01/2001 01/03/2001 31/03/2001 01/05/2001 31/05/2001 01/07/2001 31/07/2001 31/08/2001 date

Figure 9 

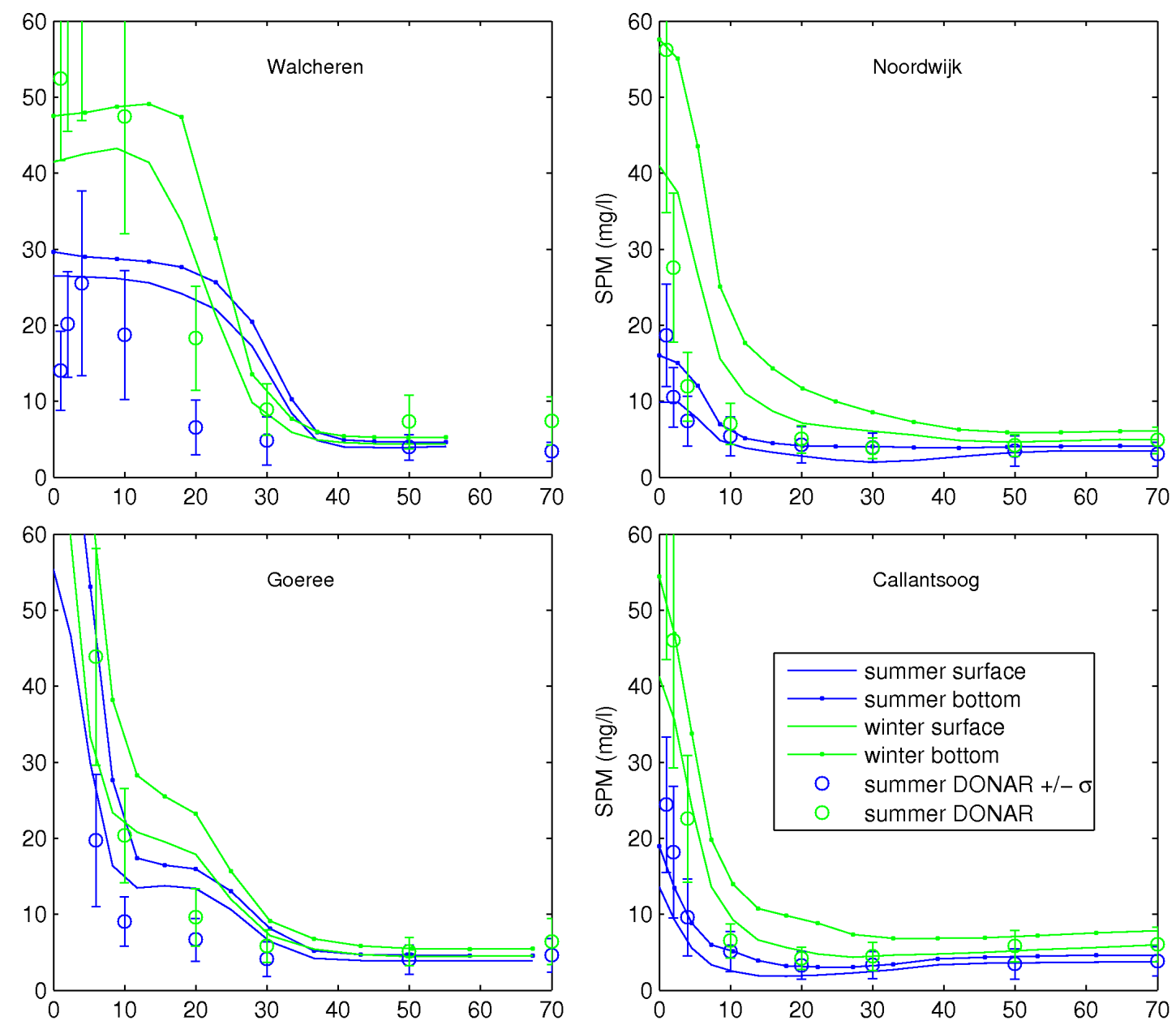

Figure 10 


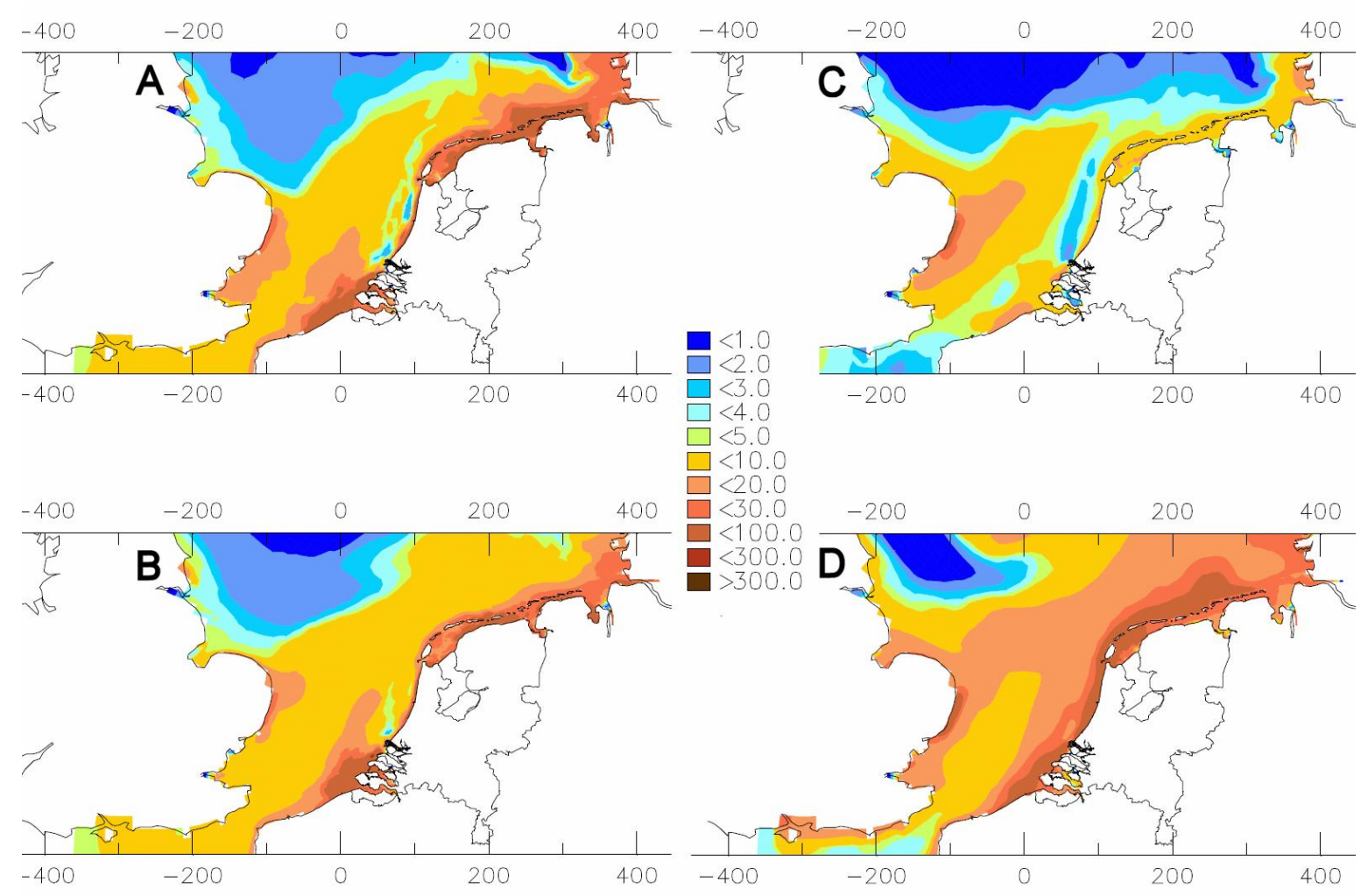

Figure 11 


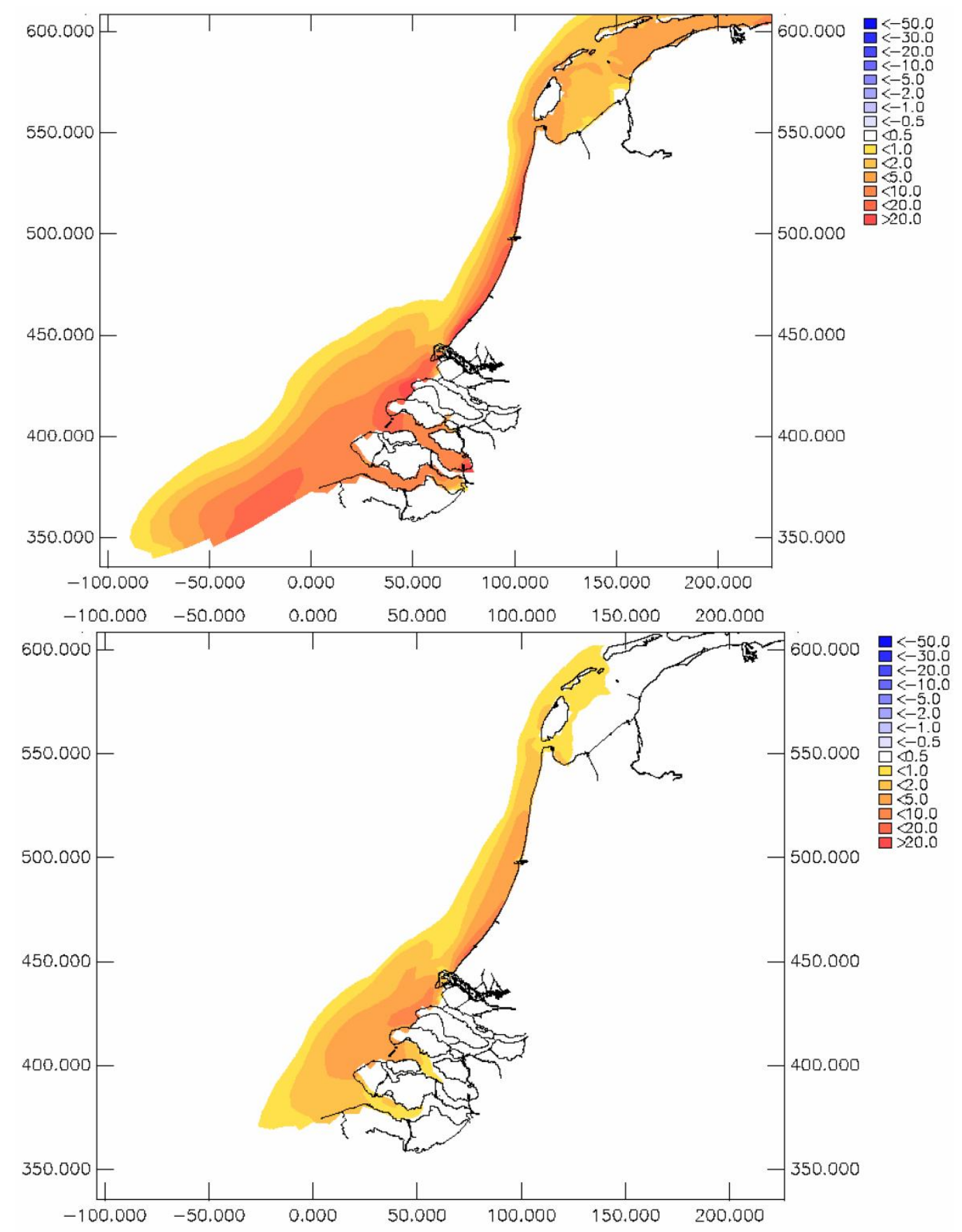

Figure 12 